\title{
PARADIGMA DA DISSEMINAÇÃO LINFÁTICA NO CARCINOMA ESPINOCELULAR DA BASE DA LÍNGUA
}

\author{
PARADIGM OF LYMPHATIC SPREAD IN SQUAMOUS CELL CARCINOMA OF THE BASE \\ OF THE TONGUE
}

\author{
Francisco S. Amorim Filho'; Josias de Andrade Sobrinho, ECBC ${ }^{1}$; \\ Abrão Rapoport, ECBC¹; Neil N. Ferreira²; Yara Juliano²
}

\begin{abstract}
RESUMO: Objetivo: O carcinoma espinocelular CEC da base da língua tem um comportamento agressivo, disseminando-se precocemente para o pescoço uni ou bilateralmente. O objetivo deste trabalho é estabelecer critérios de tratamento eletivo ou terapêutico dos linfonodos cervicais metastáticos ou não para o CEC da base de língua. Método: Estudo retrospectivo de 290 pacientes com CEC de base de língua atendidos no Departamento de Cirurgia de Cabeça e Pescoço e Otorrinolaringologia do Hospital Heliópolis, Hosphel, São Paulo, de 1997 a 2000. Após análise loco-regional, foram classificados segundo os níveis I, II, III, IV e V do estadiamento TNM da UICC (1998). Os resultados foram analisados segundo o teste do Qui-quadrado para o estudo da associação de sítios, tamanho do tumor, presença de metástases e invasão da linha média. Resultados: Houve um predomínio do sexo masculino em relação ao feminino $(9: 1)$, da $6^{\mathbf{a}}$ década, dos fumantes e etilistas $(83,8 \%)$. Quanto à disseminação linfonodal, diagnosticamos N0 (21,0\%), N1 (13,4\%), N2 (43,1\%), N3 (22,5\%) e níveis I(11,6\%), II(69,0\%), III (13,5\%), IV $(2,8 \%)$ e V $(3,1 \%)$. Quanto à invasão da linha média, não houve relação significante com o nível comprometido. Conclusão: O tratamento do CEC da base da língua deve envolver o sítio primário e os níveis de disseminação linfonodal, uni e bilateral (Rev. Col. Bras. Cir. 2006; 33(2): 79-83).
\end{abstract}

Descritores: Carcinoma de células escamosas; Língua; Neoplasias da língua; Metástase neoplásica; Pescoço.

\section{INTRODUÇÃO}

As neoplasias malignas da orofaringe e em particular da base da língua, tem um comportamento agressivo, sendo pouco freqüente em nosso meio, com evolução insidiosa, pobre sintomatologia e diagnóstico em estágios avançados em decorrência do aparecimento de metástases linfonodais no diagnóstico da doença ${ }^{1-6}$. Apresenta um predomínio do carcinoma espinocelular em $95 \%$ dos casos e ocorre com mais freqüência no homem na $6^{a}$ década de vida ${ }^{7}$.

A metástase linfonodal é muito comum nos tumores primários da base da língua devido à rica drenagem linfática desta região, constituindo-se freqüentemente (60 a 90\%) no primeiro sintoma de forma ipsilateral e de 10 a $20 \%$ bilateralmente ${ }^{2,4,5,8,9}$. O padrão de disseminação é aquele de todas as neoplasias da orofaringe, comprometendo os níveis II e III e excepcionalmente o nível IV, sendo que sua incidência aumenta com o crescimento da neoplasia primária $(\mathrm{T})^{2}$. Por outro lado, a sobrevida livre de doença é inversamente proporcional à presença de linfonodos metastáticos $(\mathrm{N})$, que determina uma maior radicalidade do procedimento cirúrgico ${ }^{5,8-10}$. Todavia, há que se considerar a existência de vairáveis neoplásicas determinantes de maior ou menor drenagem linfática como invasão da linha média, comprometimento nervoso e vascular, neoangiogênese e amplificações e detecções gênicas no CEC de base de língua.

\section{MÉTODO}

Foram analisados retrospectivamente os prontuários de 290 pacientes com carcinoma epidermóide CEC da base da língua atendidos no Departamento de Cirurgia de Cabeça e Pescoço e Otorrinolaringologia do Hospital Heliópolis, Hosphel, São Paulo, de 1977 a 2000. Todos os pacientes portadores desta neoplasia foram analisados locoregionalmente, e as cadeias linfonodais palpadas bilateralmente no pescoço, sendo registradas como níveis I(submentomandibular), II (júgulo carotídeo alto), III (júgulo carotídeo médio), IV (júgulo carotídeo baixo) e V (triângulo posterior), segundo o estadiamento TNM da UICC (1998), bem como o grau de diferenciação histológica.

Para análise dos resultados aplicou-se o teste da partição do Quiquadrado para tabelas Z x N(Cochran), com o objetivo de se estudar as associações entre os sítios e a dimensão do tumor em relação à invasão da linha média e teste do quiquadrado para tabelas Z x Z (Siegel),com a finalidade de estudar a associação entre presença de metástase e invasão da linha média.

\section{RESULTADOS}

Dos 290 pacientes portadores de carcinoma epidermóide de base de língua,encontramos uma superi-

1. Cirurgião do Departamento de Cirurgia de Cabeça e Pescoço e Otorrinolaringologia do Hospital Heliópolis, Hosphel, São Paulo.

2. Estatísticos do Departamento de Saúde Coletiva da Faculdade de Medicina da Universidade de Santo Amaro, São Paulo.

Recebido em 14/10/2005

Aceito para publicação em 10/12/2005

Conflito de interesses: nenhum

Fonte de financiamento: nenhuma

Trabalho realizado no Departamento de Cirurgia de Cabeça e Pescoço e Otorrinolaringologia do Hospital Heliópolis, Hosphel, São Paulo. 
Tabela 1 - Pacientes portadores de carcinoma epidermóide de base de língua, segundo o grau histológico da lesão primária.

\begin{tabular}{lrc}
\hline Grau histológico & \multicolumn{2}{c}{ Freqüência } \\
& N & \% \\
\hline Bem diferenciado & 40 & 13,8 \\
Moderadamente diferenciado & 152 & 52,4 \\
Pouco diferenciado & 28 & 9,6 \\
S.O.E.* & 70 & 24,1 \\
Total & $\mathbf{2 9 0}$ & $\mathbf{1 0 0}$ \\
\hline
\end{tabular}

*Sem outras especificações.

oridade masculina $(89,3 \%)$,havendo uma relação de homens e mulheres de 9:1, acometendo os pacientes predominantemente na $6 .{ }^{a}$ década de vida $(41 \%)$. A maioria dos nossos enfermos (83.8\%) fumava e bebia, havendo poucos casos (13 pacientes) em que estes hábitos estavam ausentes.

Os sintomas iniciais mais frequientes relatados por nossos pacientes foram dor à deglutição $(37.6 \%)$ e caroço no pescoço $(21.7 \%)$,sendo a média de tempo entre o sintoma inicial e o diagnóstico de até 6 meses (62\%).

O grau histológico comum à maioria dos pacientes foi o moderadamente diferenciado (152 casos) em 52,4\% (Tabela 1).

Todos os pacientes foram, classificados pelo sistema TNM (UICC, 1998), sendo encontrados 229 pacientes $(79,0 \%)$ com pescoço clinicamente positivo $(\mathrm{N}+)$ diagnosticados na primeira consulta e 61 pacientes com pescoço negativo (N0). Quando analisados o tamanho da lesão primaria e a presença de metástase cervical, obtivemos um maior número de linfonodos clinicamente positivo nos tumores mais avançados (T3 e T4) e disseminação uni ou bilateral $\left(\mathrm{N}_{2 \mathrm{c}}\right.$ e $\left.\mathrm{N}_{3}\right)$ (Tabela 2).

Das cadeias linfonodais da região cervical, a mais acometida foi o nível II em 69,0\%, nível III(13,5\%), nível I $(11,6 \%)$, nível V $(3,1 \%)$ e nível IV $(2,8 \%)$ (Tabela 3$).$

Quanto à presença dos linfonodos metástaticos cervicais e o fato da lesão primária ultrapassar ou não a linha média ,os pacientes foram assim distribuídos (Tabela 4), com evidente invasão da linha média em 192 casos (66,2\%).
Assim como na metástase regional ipsilateral, a localização dos linfonodos contralaterais, nas cadeias linfáticas do pescoço, seguiu padrão semelhante, com o nível II sendo o mais acometido e o nível $\mathrm{V}$ o menos comprometido. (Figura 1 e 2$)$.

Correlacionando as estruturas da orofaringe e sítios adjacentes, invadidos pelo tumor da base de língua, com a presença de metástase cervical clinicamente diagnosticadas, observamos que o seio piriforme, glote, tonsilar e epiglote foram os mais acometidos (Tabela 5).

Quando analisados as mesmas regiões anatômicas citadas acima ,invadidas pela lesão primaria, com a presença metástase para as cadeias inferiores (IV e V) encontramos a prega faringo-epiglótica, seio piriforme, sulco glosso-tonsilar e epiglote como as principais (Tabela 6)

\section{DISCUSSÃO}

Devido ao seu comportamento agressivo,escassa sintomatologia inicial,diagnóstico tardio da doença e prognóstico reservado na sua grande maioria, o carcinoma epidermóide de base de língua, vem sendo objeto de estudo de alguns autores ${ }^{9-11}$ interessados em diagnosticar e tratar precocemente esta neoplasia. Sua baixa incidência no terço posterior da língua (base de língua) ${ }^{12}$, faz com que os números de casos isolados destes tumores acabem sendo englobados em conjunto com os outros sítios anatômicos da orofaringe. Sua epidemiologia e comportamento não diferem dos demais tumores da orofaringe, a superioridade masculina é evidente, porem nas últimas décadas observa-se à inclusão das mulheres no consumo elevado tanto de tabaco como de álcool e uma conseqüente diminuição gradativa da proporção deste câncer entre os dois gêneros ${ }^{11,13}$. O álcool e o fumo continuam sendo os principais fatores de risco e a associação destes dois hábitos de vida estão presente na maioria dos portadores de carcinoma epidermóide de base de língua ${ }^{7,13}$. A sintomatologia inicial é escassa e silenciosa até o crescimento significativo do tumor quando então exteriorizam-se os primeiros sintomas. Em muitos casos a principal queixa que motiva o paciente a procurar o médico é a presença de linfonodos cervical.Grande parte dos enfermos chegam no ato da primeira consulta com o tumor primário avançado e com linfonodos metástaticos no pescoço $0^{2,7,14-16}$. Nossos pacientes apresentavam-se no diagnóstico inicial com o tumor

Tabela 2 - Pacientes portadores de carcinoma epidermóide de base de língua, segundo o T e o $N$.

\begin{tabular}{|c|c|c|c|c|c|c|c|c|c|c|}
\hline \multirow[t]{2}{*}{ Estriamento } & \multicolumn{2}{|c|}{ T1 } & \multicolumn{2}{|c|}{$\mathbf{T} 2$} & \multicolumn{2}{|c|}{ T3 } & \multicolumn{2}{|c|}{$\mathbf{T 4}$} & \multicolumn{2}{|c|}{ Total } \\
\hline & $\mathbf{N}$ & $\%$ & $\mathbf{N}$ & $\%$ & $\mathbf{N}$ & $\%$ & $\mathbf{N}$ & $\%$ & $\mathbf{N}$ & $\%$ \\
\hline No & 1 & 25,0 & 9 & 20,0 & 15 & 16,0 & 36 & 24,5 & 61 & 21,0 \\
\hline N1 & 1 & 25,0 & 12 & 26,7 & 11 & 11,7 & 15 & 10,2 & 39 & 13,4 \\
\hline $\mathrm{N} 2 \mathrm{a}$ & 0 & 0,0 & 8 & 17,8 & 17 & 18,1 & 16 & 10,9 & 41 & 14,1 \\
\hline $\mathrm{N} 2 \mathrm{~b}$ & 0 & 0,0 & 2 & 4,4 & 8 & 8,5 & 9 & 6,1 & 19 & 6,5 \\
\hline $\mathrm{N} 2 \mathrm{c}$ & 1 & 25,0 & 4 & 8,9 & 22 & 23,4 & 38 & 25,9 & 65 & 22,5 \\
\hline N3 & 1 & 25,0 & 10 & 22,2 & 21 & 22,3 & 33 & 22,4 & 65 & 22,5 \\
\hline Total & 4 & 100 & 45 & 100 & 94 & 100 & 147 & 100 & 290 & 100 \\
\hline
\end{tabular}


Tabela 3 - Pacientes portadores de carcinoma epidermóide de base de língua, segundo os níveis linfáticos.

\begin{tabular}{lrc}
\hline Localização & \multicolumn{2}{c}{ Freqüência } \\
& N & \% \\
\hline Nível I & 49 & 11,6 \\
Nível II & 292 & 69,0 \\
Nível III & 57 & 13,5 \\
Nível IV & 12 & 2,8 \\
Nível V & 13 & 3,1 \\
Total & $\mathbf{4 2 3}$ & $\mathbf{1 0 0}$ \\
\hline
\end{tabular}

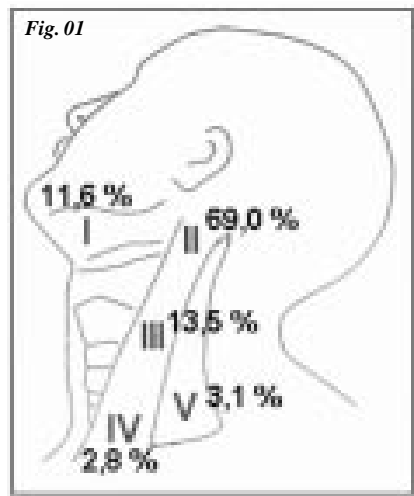

Figura 1 - Disseminação metastatica ipsilateral.

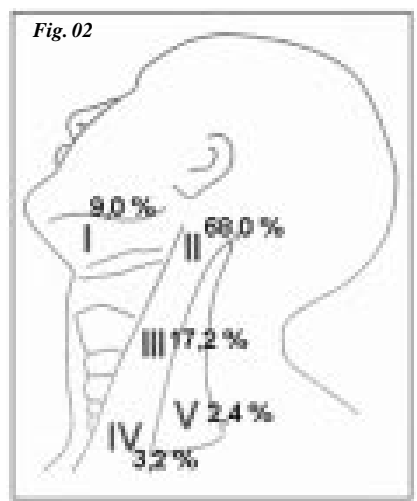

Figura 2 - Disseminação metastatica contralateral. primário em estádio avançado e pescoço clinicamente positivo em $79 \%$ dos casos, o que determina habitualmente a indicação do monobloco cirúrgico (tratamento da lesão primária e do pescoço).

Os tipos histológico mais freqüentes desta neoplasia são o moderadamente e o pouco diferenciado, estes mais agressivos, por possuírem uma característica infiltrativa quando comparados aos carcinomas bem diferenciados ${ }^{10,16}$ aspectos estes confirmados em nossa casuística.

Devido à rica rede de drenagem linfática da base de língua, o carcinoma epidermóide apresenta uma alta incidência de metástase regional, comportamento agressivo, mais freqüentes com o aumento do estadiamento $(\mathrm{T})^{2,9,15,17}$. A precoce invasão do sistema linfático cervical pode ser observada nos pacientes com pescoço clinicamente negativo e que são submetidos ao esvaziamento cervical. A incidência de linfonodos positivos, confirmados no anatomo patológico, mostra-se alta ${ }^{3,8}$.

A metástase regional quando presente pode estar associada à ruptura capsular, que tanto aumenta a incidência de recorrência da doença e desenvolvimento de metástase a distância como diminuiu a sobrevida livre de doença dos nossos pacientes.Sua presença é um indicador de prognóstico ruim $^{6,19}$. Em se tratando de orofaringe, os níveis mais acometidos são: II ,III e IV, a metástase para o nível V é uma ocorrência rara no carcinoma epidermóide do tracto aero digestivo superior $^{8-10,18-19}$, o que permite a indicação eventualmente de esvaziamento seletivo.

No nosso estudo foram constatados um maior número de metástases regionais para os níveis II (69\%), nível III $(13,5 \%)$ e nível I $(11,6 \%)$. Apesar de pouco frequiente foram achados linfonodos metástaticos no nível $\mathrm{V}$, fatos estes equivalentes aos da literatura ${ }^{10,11,17}$.

Ao correlacionarmos a presença de metástase para o pescoço e o fato da lesão primária ultrapassar ou não a linha média,observamos que na análise estatística não houve associação significante entre a presença de linfonodos metástatico regionais e o fato da lesão primária invadir a linha media da base de língua. Entretanto, chama-nos atenção a grande porcentagem de casos em que a linha media estando invadida, apresentava linfonodos metástaticos clinicamente diagnosticados. Com isto, devemos redobrar nossa atenção aos casos em que a lesão de base de língua ultrapassa a linha média e o pescoço for clinicamente negativo (casos falso-negativos), devendo manter a proposta terapêutica onde o esvaziamento bilateral merece indicação na invasão da linha média.

O carcinoma epidermóide da base de língua parece apresentar uma tendência natural de crescimento para o lado oposto ao da lesão inicial e invadir precocemente a rede linfática homolateral e contralateral,facilitada pela ausência de barreiras naturais nesta região. A metástase contralateral quando presente estava alojada com mais freqüência no níveis I,II,III sendo os níveis inferiores (IV e V) os menos acometidos, seguindo um padrão semelhante ao da metástase ipsilateral. Devemos estar atentos à possibilidade de um diagnóstico clí-

Tabela 4 - Pacientes portadores de Carcinoma Epidermóide da Base de Língua,segundo a presença de metástase regional (estádio N) e a invasão da linha média pelo tumor primário.

\begin{tabular}{|c|c|c|c|c|}
\hline \multirow{2}{*}{$\overline{\text { Estádio N }}$} & \multicolumn{4}{|c|}{ Invasão da linha média } \\
\hline & Sim & Não & Total & Proporção de Sim \\
\hline NO & 40 & 21 & 61 & $0,655738 \rightarrow 65,57 \%$ \\
\hline N1 & 27 & 12 & 39 & $0,692308 \rightarrow 69,23 \%$ \\
\hline $\mathrm{N} 2 \mathrm{a}$ & 24 & 17 & 41 & $0,585366 \rightarrow 58,54 \%$ \\
\hline $\mathrm{N} 2 \mathrm{~b}$ & 9 & 10 & 19 & $0,473684 \rightarrow 47,37 \%$ \\
\hline $\mathrm{N} 2 \mathrm{c}$ & 50 & 15 & 65 & $0,769231 \rightarrow 76,92 \%$ \\
\hline N3 & 42 & 23 & 65 & $0,646154 \rightarrow 64,62 \%$ \\
\hline Total & 192 & 98 & 290 & $0,662069 \rightarrow 66,21 \%$ \\
\hline
\end{tabular}

$p=0,02$ (não significante). 
Tabela 5- Pacientes portadores de Carcinoma Epidermóide da Base de Língua, segundo sítios comprometidos e a presença de metástase cervical.

\begin{tabular}{lcccc}
\hline Sitio & \multicolumn{2}{c}{ Metástase regional } & \\
& Sim & Não & Total & Proporção de Sim \\
\hline Valécula & 174 & 51 & 225 & $0,773333 \rightarrow 77,33 \%$ \\
Epiglote & 140 & 34 & 174 & $0,804598 \rightarrow 80,46 \%$ \\
S.glossotonsilar & 97 & 25 & 122 & $0,795082 \rightarrow 79,51 \%$ \\
Tonsila & 65 & 12 & 77 & $0,844156 \rightarrow 84,42 \%$ \\
Glote & 22 & 3 & 25 & $0,880000 \rightarrow 88,00 \%$ \\
S.Piriforme & 23 & 14 & 26 & $0,884615 \rightarrow 88,46 \%$ \\
P.Faringoepliglótica & 47 & 42 & 209 & $0,770492 \rightarrow 77,05 \%$ \\
V lingual/Corpo & 167 & $\mathbf{1 8 4}$ & $\mathbf{9 0 9}$ & $0,799043 \rightarrow 79,90 \%$ \\
Total & $\mathbf{7 3 5}$ & & $\mathbf{0 , 7 9 9 7 8 2 \rightarrow 7 9 , 9 8 \%}$ \\
\hline
\end{tabular}

$p=0,14$ não significante.

nico de linfonodo contralateral nos tumores de base de língua onde sua presença é expressiva e em geral frequente nos tumores avançados, que por sua característica infiltrativa dissemina microembolos às diversas cadeias do pescoço ${ }^{9}$.

Correlacionando as estruturas da orofaringe e sítios adjacentes, invadidos pelo carcinoma epidermóide de base de língua, com a presença de metástase cervical clinicamente diagnosticadas, observamos que a análise estatística não revelou associação significante entre o comprometimento dos sítios anatômicos e presença de metástase regional, chamando-nos atenção a grande porcentagem (80\%) de metástase presentes nestas regiões. Além disto, os sítios anatômicos invadidos pelo câncer de base de língua e as metástases nas cadeias inferiores (IV e V),não encontramos na análise estatística associação significante entre estes dois dados. Porém, observamos que a glote quando comprometida pelo tumor de base de língua, não apresentava metástase para os níveis IV e V,e os demais sítios apresentaram metástase para estes níveis com valores expressivos $(11 \%)$ quando comparados aos dados da literatura ${ }^{9,16-18}$, o que dificulta a padronização terapêutica.

O tratamento ideal para tumores de base de língua gera inúmeras controvérsias, principalmente nos casos avan- çados, para os quais a associação de modalidades terapêuticas diferentes parece ter um melhor controle da doença. $\mathrm{O}$ tratamento deve ser direcionado tanto a lesão primária como para as possíveis metástases regionais. A possibilidade de um tratamento mais radical com esvaziamento funcional poderá ser efetuada nos casos em que a lesão primária segue em direção à hipofaringe (prega faringoepiglótica e seio piriforme), mesmo diante de um pescoço clinicamente negativo, pois entre os nossos pacientes, encontramos,ao exame histopatológico, uma alta incidência de metástases nos níveis IV e $\mathrm{V}$ quando o referido sitio estava invadido. $\mathrm{O}$ tratamento com esvaziamento seletivo, para o pescoço, poderá ser adotado nos casos em que o tumor de base de língua se dirigir à glote e o pescoço for clinicamente negativo, justificando com isto tal procedimento.

Devemos lembrar que independente destes achados ,o carcinoma epidermóide de base de língua é agressivo e metastatiza para o pescoço com freqüência, sendo desejável que se proceda, antes de tudo, uma exploração cuidadosa das cadeias linfáticas do pescoço homolateral ou contralateral, principalmente nos tumores de base de língua que ultrapassam a linha média, permitindo eventuais mudanças terapêuticas trans-operatórias.

Tabela 6 - Distribuição dos pacientes portadores de Carcinoma Epidermóide da Base de Língua, segundo sítios comprometidos X metástase cervicais nas cadeias inferiores.

\begin{tabular}{|c|c|c|c|c|}
\hline \multirow[t]{2}{*}{ Sítios Anatômico } & \multicolumn{4}{|c|}{ Metástase } \\
\hline & Nível IV,V & Nível I,II,III & Total & Proporção de Metástase \\
\hline Valécula & 18 & 156 & 174 & $0,103448 \rightarrow 10,34 \%$ \\
\hline Epiglote & 16 & 124 & 140 & $0,114286 \rightarrow \quad 11,43 \%$ \\
\hline S.Glossotonsilar & 12 & 85 & 97 & $0,123711 \rightarrow \quad 12,37 \%$ \\
\hline Tonsila & 7 & 58 & 65 & $0,107692 \rightarrow \quad 10,77 \%$ \\
\hline Glote & 0 & 22 & 22 & $0,000000 \rightarrow \quad 0,00 \%$ \\
\hline S.piriforme & 4 & $\overline{19}$ & 23 & $0,173913 \rightarrow \quad 17,39 \%$ \\
\hline P.Faringoepiglotica & 9 & 38 & 47 & $0,191489 \rightarrow \quad 19,15 \%$ \\
\hline V.Lingual & 16 & 151 & 167 & $0,095808 \rightarrow \quad 9,58 \%$ \\
\hline Total & 82 & 653 & 735 & $0,111565 \rightarrow 1 \quad 1,16 \%$ \\
\hline
\end{tabular}

$p=0,4$ não siginificante. 


\begin{abstract}
Background; Squamous cell carcinoma (SCC) of the base of the tongue is an asymptomatic and aggressive neoplasia, spreading uni or bilateral routinely to the neck. The objective is to establish the elective or therapeutic therapy for metastatic neck nodes for SCC of base of the tongue. Method: Through a retrospective analysis of 290 files of patients with SCC of the base of the tongue from Departamento de Cirurgia de Cabeça e Pescoço e Otorrinolaringologia do Hospital Heliópolis, Hosphel, São Paulo de 1997 a 2000, after a locoregional assessment, the patients were classified as levels I, II, III, IV, V from TNM staging of UICC (1998). The results were evaluated through Chi Square Test for the association of site, tumor size, metastases and medium line invasion. Results: The relation between male and female were 9:1, the $6^{\text {th }}$ decade and alcohol, tobacco were predominant (83.8\%). Concerning lymph node spreading, NO (21.0\%), N1 (13.4\%), N2 (43.1\%), N3(22.5\%) and level I (11.6\%), II (69.0\%), III (13.5\%), IV (2.8\%) and V (3.1\%). About concerning medium line spread, it was not a significant relation with affected level. Conclusion: The treatment of SCC of the base of the tongue involve the primary lesion and the lymph node spread to uni and bilateral neck level.
\end{abstract}

Key words: Carcinoma, squamous cell; Tongue; Tongue neoplasms; Neoplasm metastasis; Neck.

\section{REFERÊNCIAS}

1. Civantos FJ, Goodwin Jr WJ. Cancer of the oropharynx. In: Myers EN, Suen JY, editors. Cancer of the head and neck. Philadelphia. WB Saunders; 1996. p.

2. Kurtulmaz SY, Erkal HS, Serin M, Elhan AH, Cakmak A. Squamous cell carcinomas of the head and neck :descriptive analysis of 1293 cases. J Laryngol Otol. 1997; 111(6):531-5.

3. Adamns GL. Cancer of the oropharynx. In: McQuarrie, DG, Adamns GL, Shons AR, Browne GA, editors. Head and neck cancer-clinical decisions and management principles. St. Louis: Mosby; 1986. p.

4. Dicker A, Harrison LB, Picken CA, Sessions RB, O’Malley BB. Oropharyngeal cancer. In: Harrison LB, Sessions RB, Ki Hong W, editors. Head and neck cancer: a multidisciplinary approach. Philadelphia: Lippincott-Raven, 1999. p.

5. Kraus DH, Vastola AP, Huvos AG, Spiro RH. Surgical management of squamous cell carcinoma of the base of tongue. Am J Surg. 1993; 166(4):384-8.

6. Johnson JT, Myers EN, Bedetti CD, Barnes EL, Schramm VL Jr, Thearle PB. Cervical lymph node metastases. Incidence and implications of extracapsular carcinoma. Arch Otolaryngol. 1985; 111(8):534-7.

7. Johansen LV, Grau C, Overgaard J. Squamous cell carcinoma of the oropharynx - an analysis of treatment results in 289 consecutive patients. Acta Oncol. 2000; 39(8):985-94.

8. Jose J, Coatesworth AP, Johnston C, MacLennan K. Cervical node metastases in oropharyngeal squamous cell carcinoma: prospective analysis of prevalence and distribution. J Laryngol Otol. 2002; 116(11):925-8.

9. Foote RL, Olsen KD, Davis DL, Buskirk SJ, Stanley RJ, Kunselman SJ, Schaid DJ, DeSanto LW. Base of tongue carcinoma: patterns of failure and predictors of recurrence after surgery alone. Head Neck.1993; 15(4):300-7.

10. Shah JP. Patterns of cervical lymph node metastasis from squamous carcinomas of the upper aerodigestive tract. Am J Surg. 1990; 160(4):405-9.

11. Amorim Filho FS, Andrade Sobrinho J, Rapoport A, Carvalho MB, Novo NF, Juliano Y. Estudo de variáveis demográficas, ocupacionais e co-carcinogenéticas no carcinoma espinocelular da base de língua nas mulheres. Rev Bras Otorrinolaringol. 2003; 69(4):472-8.
12. Seikaly H, Rassekh CH. Oropharyngeal cancer. In: Bailey BJ, Calhoum KH, Deskin, RW, Johnson JT, Kohut RF, Pillsbury $3^{\text {rd }}$ HC, Tardy Jr, ME. Head and neck surgery: otolaryngology. $2^{\text {nd }}$ ed. Philadelphia: Lippincott-Raven; 1998. p.

13. Sanderson RJ, de Boer MF, Damhuis RA, Meeuwis CA, Knegt PP. The influence of alcohol and smoking on the incidence of oral and oropharyngeal cancer in women. Clin Otolaryngol Allied Sci. 1997; 22(5):444-8.

14. Perlmutter MA, Johnson JT, Snyderman CH, Cano ER, Myers EN. Functional outcomes after treatment of squamous cell carcinoma of the base of tongue.Arch Otolaryngol Head Neck Surg 2002; 128:887-891.

15. Nason RW, Anderson BJ, Gujrathi DS, Abdoh AA, Cooke RC. A retrospective comparison of treatment outcome in the posterior and anterior tongue.Am J Surg. 1996; 172(6):665-70.

16. Kowalski LP, Franco EL, Torloni H, Fava AS, Andrade Sobrinho J, Ramos G, Oliveira BV, Curado MP. Lateness of diagnosis of oral and oropharyngeal carcinoma: factors related to tumour, the patients and health professionals. Eur J Cancer B Oral Oncol. 1994; 30B(3):167-73.

17. Li XM, Wei WI, Guo XF, Yuen PW, Lam LK. Cervical lymph node metastatic patterns of squamous carcinomas in the upper aerodigestive tract. J Laryngol Otol. 1996; 110(10):937-41.

18. Davidson BJ, Kulkarny V, Delacure MD, Shah JP. Posterior triangle metastases of squamous cell carcinoma of the upper aerodigestive tract. Am J Surg. 1993; 166(4):395-8.

19. Lindberg R. Distribution of cervical lymph node metastases from squamous cell carcinoma of the upper respiratory and digestive tracts. Cancer. 1972:29(6):1446-9.

Como citar este artigo:

Amorim FS, Sobrinho JA, Rapoport A, Ferreira NN, Juliano Y. Paradigma da disseminação ganglionar no carcinoma espinocelular da base da língua. Rev Col Bras Cir. [serial on Internet]. 2006 Mar-Apr; 33(2). Available from URL: http://www.scielo.br/rcbc

Endereço para correspondência:

Prof. Dr. Abrão Rapoport

Rua Iramaia, $n^{\circ} 136$ - Jd. Europa

CEP: 01450 - 020 - São Paulo - SP

E-mail: arapopor@terra.com.br 